\section{Genotipo y fenotipo de la enzima tiopurina metiltransferasa en población chilena}

\author{
ANDRÉS JORQUERA ${ }^{1,2}$, SANDRA SOLARI ${ }^{3}$, \\ VALESKA VOLLRATH ${ }^{1, \mathrm{a}}$, IRENE GUERRA ${ }^{3, \mathrm{~b}}$, JOSÉ CHIANALE ${ }^{1}$, \\ COLOMBA COFRÉ $^{4,5}$, ALEXIS KALERGIS ${ }^{6}$, PATRICIO IBÁÑEZ ${ }^{1}$, \\ SUSAN BUENO ${ }^{6}$, MANUEL ÁLVAREZ-LOBOS ${ }^{1}$
}

\section{Phenotype and genotype of thiopurine methyltransferase in Chilean individuals}

Background: Thiopurines (azathioprine and 6-mercaptopurine) are highly effective medications but with potential adverse effects. Thiopurine methyltransferase (TMPT) is the key enzyme in their pharmacokinetics and is genetically regulated. A low activity of TPMT is associated with myelotoxicity. The genotype and enzyme activity can vary by ethnicity. Aim: To study the activity and genotype of TPMT in a group of Chilean subjects. Material and Methods: In 200 healthy adult blood donors, TPMT activity was determined by high performance liquid chromatography (HPLC). Deficient, low, normal or high levels were defined when enzymatic activity was $\leq 5,6-24,25-55$ and $\geq 56 \mathrm{nmol} / \mathrm{grHb} / \mathrm{h}$, respectively. Genotyping of TPMT $\left({ }^{*} 1\right.$, $\left.{ }^{\star} 2,{ }^{*} 3 A,{ }^{*} 3 B,{ }^{*} 3 C\right)$ was performed by PCR. Results: Seventy seven women (38.5\%) and 123 men (61.5\%), with an average age of 34.9 years were studied. Eighteen subjects (9\%) had a low enzymatic activity, 178 (89\%) had normal activity, 4 (2\%) had high activity and no genotype deficient subjects were identified. The wild type genotype $\left({ }^{*} 1\right)$ was found in $184(92 \%)$ individuals and $16(8 \%)$ were heterozygous for the variants: ${ }^{\star} 2(n=2),{ }^{\star} 3 A(n=13)$ and ${ }^{\star} 3 C(n=1)$. No homozygous subjects for these variants were identified. Wild type genotype had an increased enzymatic activity $(40.8 \pm 7.2 \mathrm{nmol} / \mathrm{gHb} / \mathrm{h})$ compared to heterozygous group $(21.2 \pm 3 \mathrm{nmol} /$ $\mathrm{gHb} / \mathrm{h} ; \mathrm{p}<0.001)$. Conclusions: Less than $10 \%$ of a Chilean population sample has a low enzymatic activity or allelic variants in the TPMT gene, supporting the use of thiopurines according to international recommendations.

(Rev Med Chile 2012; 140: 889-895).

Key words: Drug hypersensitivity; Chile; Gentype; TPMT deficiency; Phenotype.

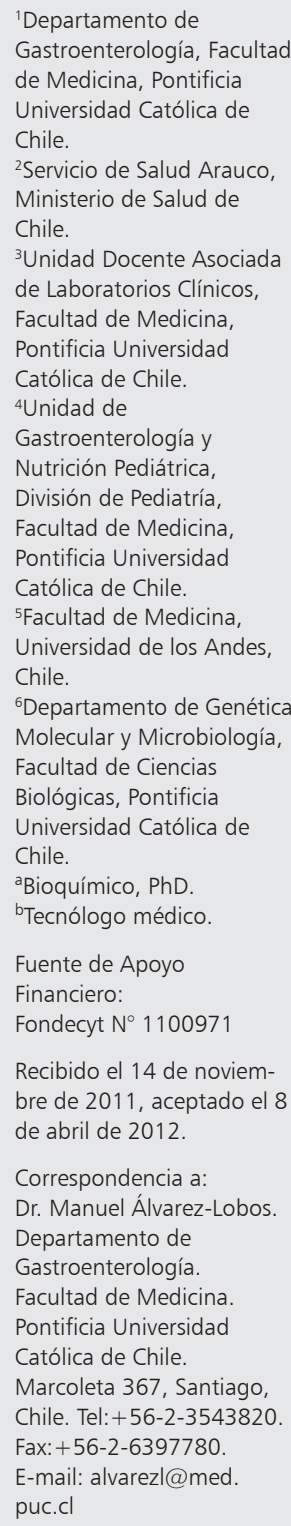

L os tiopurínicos, azatioprina (AZA) y 6-mercaptopurina (6-MP), son fármacos efectivos y ampliamente utilizados en diversas patologías complejas que presentan una prevalencia creciente como son las enfermedades inflamatorias intestinales (EII), hepatitis autoinmune, trasplante de riñón o hígado, lupus eritematoso sistémico, esclerosis múltiple o leucemia linfática aguda entre otras ${ }^{1-9}$.

Sin embargo, existe una variabilidad individual en la efectividad y pueden producir importantes efectos adversos ${ }^{1-3,10}$. Esto se debe principalmente a la dosis utilizada y a una susceptibilidad individual $^{1-3,10}$. En las dosis utilizadas en pacientes con EII (2,0-3,0 mg/kg/día), los efectos adversos se observan en 10 a $20 \%$ de los pacientes ${ }^{1-3,10}$, los que incluyen mielotoxicidad, molestias gastrointestinales, toxicidad hepática o pancreatitis. Entre estas, la mielotoxicidad es la más importante, la que puede llevar a una leucopenia potencialmente mortal $^{9,10}$. 
La farmacocinética está mediada por varias enzimas, con dos vías principales: una catabólica que inactiva el fármaco, y una vía anabólica de síntesis de nucleótidos de 6 tioguanina (6-TG), los cuales ejercen el efecto farmacológico (Figura 1) ) $^{1-3,10}$. La enzima clave en su metabolismo es la tiopurina metiltransferasa (TPMT). La TMPT es una enzima citosólica que cataliza la metilación de 6-mercaptopurina (6-MP) a 6-metilmercaptopurina, un metabolito inactivo ${ }^{1,10-15}$. Una baja actividad enzimática desviaría preferentemente el metabolismo de 6-MP a una mayor producción de 6-TG, responsables del efecto inmunosupresor y mielosupresor, mediante el bloqueo de la síntesis de ADN e inducción de apoptosis

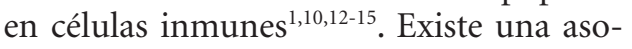
ciación entre disminución de la actividad de la TPMT y toxicidad medular mediada por $6-\mathrm{TG}^{1,10,15}$.

La actividad enzimática de la TPMT (fenotipo) está condicionada por polimorfismos genéticos (genotipo) de nucleótido único (SNP) $)^{10,12-15}$. Sobre 20 diferentes variantes alélicas existen para este gen, localizado en el brazo corto del cromosoma $6^{12}$. El genotipo wild type de la TPMT $\left({ }^{\star} 1\right)$ se observa en aproximadamente $90 \%{ }^{10,12-15}$. Las variantes alélicas mutantes más frecuentes en la población son la ${ }^{\star} 2,{ }^{\star} 3 \mathrm{~A}{ }^{\star} 3 \mathrm{~B}$ y ${ }^{\star} 3 \mathrm{C}$. (Tabla 1) $)^{10,12-15}$. Estas variantes explican 95\% de los casos de actividad enzimática baja o deficiente ${ }^{14}$. En el fenotipo, $88 \%$ de la población caucásica tiene actividad normal o elevada, $11 \%$ tienen actividad baja (50\% de la actividad enzimática normal) y menos de $1 \%$ tienen actividad deficiente $e^{12,15,16}$. Los individuos con baja o deficiente actividad de TPMT están expuestos a un riesgo mayor de toxicidad ${ }^{1,10,12-15}$. Sujetos con actividad alta pueden requerir dosis mayores de tiopurínicos para alcanzar el efecto terapéutico ${ }^{1,10,15}$.

Existen diferencias étnicas en las frecuencias de variantes genéticas para la $\mathrm{TPMT}^{10,16}$. Esto ha sido escasamente estudiado en población latinoamericana mestiza, sin una adecuada descripción del grado de mezcla hispánica y amerindia ${ }^{17,18}$. En Chile no existen recomendaciones basadas en estudios locales de correlación genotipo-

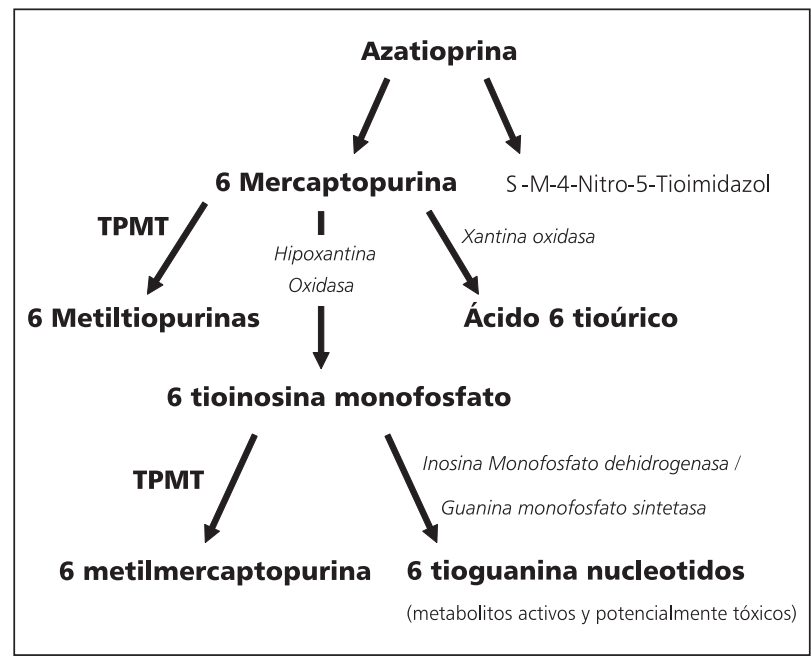

Figura 1. Farmacocinética de los tiopurínicos. TPMT: tiopurina metiltransferasa. Adaptado de Ho et $\mathrm{al}^{10}$.

Tabla 1. Polimorfismos de las variantes del gen de la TPMT

\begin{tabular}{|clcc|}
\hline Exón & Alelo & SNP & $\begin{array}{c}\text { Cambio } \\
\text { aminoacídico }\end{array}$ \\
\hline 5 & TPMT $^{*} 2$ & G238C & Ala80Pro \\
7 y 10 & TPMT $^{*} 3 \mathrm{~A}$ & G460A y A719G & Ala154Thr y Tyr240Cis \\
\hline 7 & TPMT*3B $^{*}$ & G460A & Ala154Thr \\
\hline 10 & TPMT $* 3 C^{*}$ & A719G & Tyr240Cis \\
\hline
\end{tabular}

fenotipo, lo que puede contribuir a una dosificación insuficiente de tiopurínicos por largos períodos, por lo que conocer su estado permitirá dar recomendaciones más eficientes y seguras.

El objetivo del estudio es realizar una genotipificación y fenotipificación de la enzima TPMT en población adulta promedio chilena de origen hispano.

\section{Materiales y Métodos}

\section{Población}

Bajo consentimiento informado se reclutaron individuos chilenos sanos, mayores de 18 años, no relacionados, con ambos apellidos hispanos, que asistieron consecutivamente como donantes del banco de sangre al Hospital Clínico de la Pontificia Universidad Católica de Chile. Mediante la distribución de los grupos sanguíneos (AB-0), se determinó el índice de mezcla amerindia a 
través de la prevalencia del grupo sanguíneo 0 según el método de Bernstein ${ }^{19-21}$. La distribución de grupos sanguíneos de la población estudiada fué la siguiente: Grupo A: 28,7\%, grupo B: 10,7\%, grupo AB: 2,0\% y grupo O: 58,4\% y el índice de mezcla amerindia de nuestra población fue de 0,33 el cual corresponde a un estrato socioeconómico medio de la ciudad de Santiago de Chile ${ }^{19-20}$.

Se extrajo una muestra de sangre de $10 \mathrm{ml}$ en EDTA, de la cual se aislaron glóbulos rojos para la determinación de la actividad enzimática ${ }^{23,24}$. Otra muestra de $5 \mathrm{ml}$ de sangre en EDTA se utilizó para extracción de ADN y genotipificación. El estudio fue aprobado por el comité de ética de nuestra institución.

\section{Determinación de la actividad enzimática de TPMT}

Preparación del lisado y análisis de hemoglobina

Los tubos con sangre fueron centrifugadas a $1.400 \mathrm{~g}$ por $5 \mathrm{~min}$. El plasma fue removido y los glóbulos rojos (GR) lavados con $2 \mathrm{ml}$ de suero fisiológico, mezclados por inversión y luego centrifugados a $1.400 \mathrm{~g}$ por $5 \mathrm{~min}$. Los GR se guardaron a $-70^{\circ} \mathrm{C}$ hasta el análisis, en la que se lisaron los GR y se determinó la concentración de hemoglobina ( $\mathrm{Hb})$ mediante técnicas previamente descritas ${ }^{22,23}$.

Incubación y análisis por cromatografía líquida de alta resolución (HPLC)

La actividad enzimática de la TPMT fue determinada utilizando 6-TG como sustrato y se midió el producto de la reacción, 6-metiltioguanina (6MTG), mediante cromatografía líquida de alta resolución (HPLC) ${ }^{22,23}$. Se preparó una mezcla de incubación, con una concentración de $600 \mu \mathrm{mol} / \mathrm{L}$ de 6-TG (Sigma-Aldrich) y $80 \mu \mathrm{mol} / \mathrm{S}$-adenosilL-metionina (Fluka Biochemica) en 0,1 mol/L de buffer de fosfato de potasio $(\mathrm{pH} \mathrm{7,4)}$, la cual se alicuotó $(0,5 \mathrm{~mL})$ en tubos Eppendorf. Después de incubar los tubos Eppendorf por $5 \mathrm{~min}$ a $37^{\circ} \mathrm{C}$, se agregó $0,2 \mathrm{ml}$ de los GR lisados y se incubó a $37^{\circ} \mathrm{C}$ por una hora. Para detener la reacción enzimática, los tubos se incubaron por $10 \mathrm{~min}$ a $85^{\circ} \mathrm{C}$. Los tubos fueron centrifugados a $1.400 \mathrm{~g}$ por $5 \mathrm{~min}$ y el sobrenadante se inyectó al cromatógrafo ${ }^{22,23}$.

El análisis por HPLC se realizó utilizando equipamiento de Waters Corporation, Milford, MA, USA (Bomba 600, muestreador automático 717 , volumen de inyección de $20 \mu \mathrm{L}$, detector de fluorescencia 474, excitación a 310 nm y emisión a 390 nm; columna Zorbax Eclypse XDB-C18, (Agilent Technologies, USA). La fase móvil (flujo de $1,5 \mathrm{ml} / \mathrm{min}$ isocrático) estaba compuesta de buffer fosfato $50 \mathrm{mmol} / \mathrm{L}$, acetonitrilo, tetrahidrofurano (91:5:4, v/v, a un pH 6,2). Se utilizó un punto de 0,44 nmol (80 ng) de 6-MTG para estandarizar el ensayo. Un blanco del estándar $(0,2 \mathrm{~mL}$ de suero fisiológico) fue realizado para corregir la producción no enzimática de 6-MTG ${ }^{22,23}$.

El nivel de actividad de TPMT fue calculada estimando el área bajo la curva del pico de 6-MTG. Este método ha sido validado y se categorizaron los valores expresados en nmol 6-MTG por gramo de $\mathrm{Hb}$ por hora (nmol/gHb/h) en: actividad deficiente $\leq 5$, actividad baja 6-24, actividad normal 25-55 y actividad alta $\geq 56^{22,23}$.

\section{Determinación genética de la TPMT}

Las variantes alélicas del gen TPMT que se estudiaron fueron aquellas descritas previamente como más frecuentes como son: la TPMT ${ }^{\star} 2$, mutación G238C (exón 5); la TPMT*3A, tiene dos mutaciones simultáneas, G460A (exon5) y A719G (exón 10); la TPMT³B, mutación G460A (exón 7); y la TPMT³C, mutación A719G (exón 10) ${ }^{11}$. Se aisló ADN genómico desde sangre periférica utilizando el kit Wizard Genomic (Promega) según instrucciones del fabricante. La genotipificación se realizó mediante técnica de RFLP-PCR ${ }^{12}$. Los partidores específicos para los polimorfismos de cada gen, así como los fragmentos de digestión de cada alelo se detallan en la Tabla 2. Para los polimorfismos de los exones 7 y 10 existen enzimas de restricción comerciales. Sin embargo, para el polimorfismo del exón 5 (G238C) no hay enzima de restricción comercial y se diseñó uno de los partidores con una sustitución de una base para generar la secuencia de reconocimiento de una ER. Este cambio no altera la unión del partidor y permite obtener un producto amplificado con la secuencia de reconocimiento para la ER (la base substituida está subrayada en Tabla 2). La amplificación mediante PCR y la genotipificación de los genes de TPMT estudiados, se realizó de acuerdo a lo previamente descrito ${ }^{12}$.

\section{Análisis estadístico}

Se estimó que esperando encontrar una prevalencia de actividad enzimática baja de la TPMT (heterocigotos) de 11\% y actividad nula de 0,3\%, 
Tabla 2. Partidores y productos de digestión de las enzimas de restricción utilizados en la genotipificación del gen TPMT

\begin{tabular}{|c|c|c|}
\hline Exón (mutación) & Partidores & Productos digeridos (pares de bases) \\
\hline \multirow[t]{2}{*}{ (G238C) } & F:5'ATGCTTACTCTAATATAACCCTC 3' & Wt $(80,20) ;$ Mut $(100)^{a}$ \\
\hline & R: 5 TACACTGTGTCCCCGGGCTG'3 & \\
\hline \multirow[t]{2}{*}{$7 \quad(\mathrm{G} 460 \mathrm{~A})$} & F: 5' AAGTACCAGCATGCACCATG 3' & Wt $(135,125) ;$ Mut $(260)^{b}$ \\
\hline & R: 5' AAACTCATAGAAGTCTAAGCTG 3' & \\
\hline \multirow[t]{2}{*}{ (A719G) } & F: 5' ATGCAATATACGTTGTCTTGAG 3' & Wt (185); Mut $(97,88)^{c}$ \\
\hline & R: 5' CATTACATTTTCAGGCTTTAGC 3' & \\
\hline
\end{tabular}

Wt: Wild type o silvestre; Mut: Mutado. a: La enzima de restricción utilizada fue Satl (Fnu4HI), de Fermentas International Inc. b: La enzima de restricción utilizada fue Mwol (HpyF10VI), de Fermentas International Inc. c: La enzima de restricción utilizada fue Xmil (Accl), de Fermentas International Inc.

similar a lo descrito en otras poblaciones caucásicas $^{12}$, con un intervalo de confianza de $95 \%$ y un poder de $80 \%$ se requiere un mínimo de 177 individuos.

El análisis comparativo del nivel de actividad enzimática se realizó mediante T test. Las diferencias se consideraron significativas con un valor de $\mathrm{p}<0,05$.

\section{Resultados}

\section{Características de la población}

Se reclutaron 200 voluntarios sanos donantes de sangre, $77(38,5 \%)$ mujeres y $123(61,5 \%)$ hombres, edad promedio 34,9 años, rango de 18 a 61 años, con ambos apellidos hispánicos.

\section{Actividad enzimática (HPLC)}

El rango de distribución de la actividad enzimática fue de 16,87 a $71,51 \mathrm{nmol} / \mathrm{gHb} / \mathrm{h}$. Los intervalos de distribución de nuestra población se muestran en la Tabla 3. No hubo correlación entre la actividad enzimática y la edad o el sexo. (T test: $\mathrm{p}=0,84$ y $\mathrm{p}=0,76$ ).

\section{Genotipificación de la TPMT}

Frecuencia de los polimorfismos de la TPMT

De los 200 individuos estudiados, 184 (92\%) resultaron ser wild type $\left({ }^{\star} 1\right)$ y 16 individuos $(8 \%)$ resultaron heterocigotos para alguna de las variantes genéticas estudiadas. No se encontraron individuos homocigotos para variantes genéticas.
La variante alélica más frecuente fue la $* 3 \mathrm{~A}(13$ individuos), luego la ${ }^{\star} 2$ (dos individuos) y uno con la variante ${ }^{\star} 3 \mathrm{C}$. La distribución está en concordancia con la predicción de Hardy-Weinberg para herencia autosómica codominante para un par de alelos, uno con baja y el otro con normal-alta actividad, con frecuencias de 0,04 y 0,96 , respectivamente, similar a lo descrito previamente en otras poblaciones que presentan frecuencias de $0,05 \mathrm{y}$ 0,94 respectivamente $^{12}$. Las frecuencias alélicas y genotípicas se muestran en la Tabla 4.

Tabla 3. Intervalos de distribución de la actividad enzimática de la TPMT en una muestra de población chilena $(n=200)$

\begin{tabular}{|c|c|c|}
\hline \multicolumn{2}{|c|}{$\begin{array}{l}\text { Intervalo actividad } \\
\text { (nmol/gHb/h) }\end{array}$} & $\begin{array}{c}\text { n de individuos } \\
(\%)\end{array}$ \\
\hline Deficiente & $(\leq 5)$ & 0 \\
\hline Baja & $(6-24)$ & 18 (9) \\
\hline Normal & $(25-55)$ & 178 (89) \\
\hline Alta & $(\geq 56)$ & $4 \quad(2)$ \\
\hline
\end{tabular}

Tabla 4. Distribución genotípica del gen TPMT en población chilena $(n=200)$

\begin{tabular}{|ccccc|}
\hline \multicolumn{5}{|c|}{ Frecuencia genotípica, $\mathbf{n}(\mathbf{\%})$} \\
$\boldsymbol{*}_{\mathbf{1}} \boldsymbol{*}^{\mathbf{1}}$ & $\boldsymbol{*}_{\mathbf{1}} \mathbf{}_{\mathbf{2}}$ & $\boldsymbol{*}_{\mathbf{1}} / \mathbf{*} \mathbf{A}$ & ${ }^{*} \mathbf{1} / \mathbf{3 B}$ & ${ }^{*} \mathbf{1} / \mathbf{3 A}$ \\
$184(92)$ & $2(1)$ & $13(6,5)$ & 0 & $1(0,5)$ \\
\hline
\end{tabular}

*1: Wild type o silvestre del gen de la TPMT. *2, *3A, *3B, *3C: Variantes génicas del gen de la TPM. 


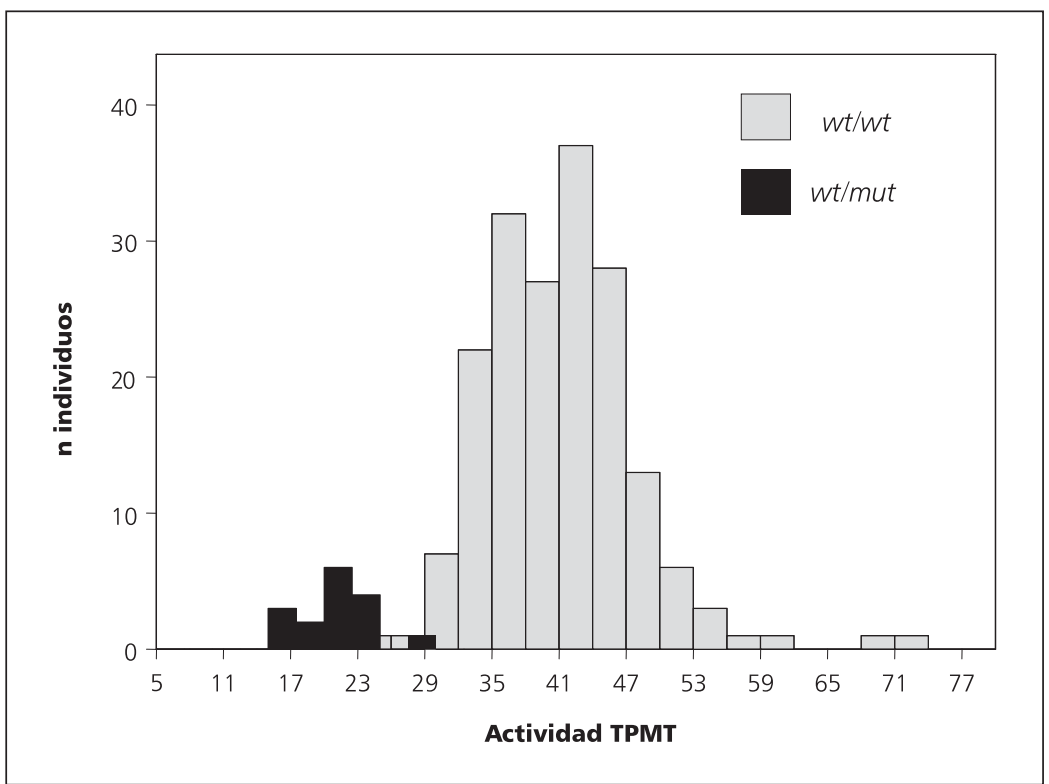

Figura 2. Distribución de la actividad enzimática en población chilena según el genotipo de la TPMT. wt: wild type (silvestre); mut: mutado.

\section{Correlación genotipo-fenotipo de los} polimorfismos de la TPMT

Los wild type $(\mathrm{n}=184)$ tuvieron una actividad media de $40,8 \pm 7,2 \mathrm{nmol} / \mathrm{grHb} / \mathrm{h}$ y en el grupo de heterocigotos $(\mathrm{n}=16)$ la actividad media fue 21,2 $\pm 3 \mathrm{nmol} / \mathrm{gHb} / \mathrm{h}$ y esta diferencia fue estadísticamente significativa ( $\mathrm{t}$ test: $\mathrm{p}<0,001$ ).

Hubo tres individuos wild type para las mutaciones analizadas que tuvieron un nivel de actividad bajo el punto de corte. Además hubo un individuo con genotipo heterocigoto (3A) que tuvo una actividad normal de $28,2 \mathrm{nmol} /$ $\mathrm{gHb} / \mathrm{h}$ (Figura 2). La distribución de la actividad enzimática en nuestra muestra sigue un patrón bimodal, descrito en otras poblaciones, según su genotipo $^{12,14,17,18,24}$.

\section{Discusión}

El presente trabajo corresponde al primer estudio de correlación genotipo-fenotipo para la TPMT en población chilena hispana y nuestros resultados indican que se comporta en forma similar a otras poblaciones occidentales, tanto en frecuencia y distribución de los genotipos más frecuentes como en la expresión fenotípica de la TPMT $^{14,24-26}$. Esta población de donantes voluntarios de banco de sangre representaría, según su índice de mezcla amerindia de 0,33 , a un estrato socioeconómico medio de Santiago de Chile ${ }^{19,20}$, por lo que los resultados son extrapolables a la mayoría de la población chilena.

Desde el punto de vista fenotípico, $91 \%$ de la población chilena presenta actividad de TPMT enzimática normal o alta, y $9 \%$ de la población presenta actividad baja. En relación al genotipo de TPMT podemos decir que $92 \%$ de la población chilena es wild type y $8 \%$ de la población es heterocigoto para cualquiera de las variantes analizadas. Nuestros resultados tienen relevancia clínica, y respalda el uso de los tiopurínicos de una manera similar a las poblaciones caucásicas, con dosis adecuadas según las guías de tratamiento internacionales establecidas ${ }^{25-27}$. La genotipificación y fenotipificación de la TPMT son de una relativa simple implementación, lo que permitiría alcanzar dosis efectivas y seguras de los tiopurínicos en un plazo breve de acuerdo al resultado de cada individuo. En pacientes chilenos con enfermedad inflamatoria intestinal homocigotos para las variantes genéticas o con actividad deficiente $(<5 \mathrm{nmol} / \mathrm{gHb} / \mathrm{h})$ se deben evitar los tiopurínicos y usar otros inmunomoduladores o indicar minidosis (2,5-5 mg/d de azatioprina). En pacientes heterocigotos para las variantes genéticas, o con baja actividad, se recomienda iniciar la azatioprina a dosis bajas de $0,5 \mathrm{mg} / \mathrm{kg} /$ día, hasta llegar a dosis 
máxima de $1 \mathrm{mg} / \mathrm{kg} /$ día en el plazo de un mes ${ }^{10,25,27}$. Los pacientes wild type con actividad normal (25$55 \mathrm{nmol} / \mathrm{gHb} / \mathrm{h}$ ) podrían iniciar dosis de 2-2,5 mg/ $\mathrm{kg} / \mathrm{d}$ de azatioprina o estas dosis alcanzarse en un plazo de pocas semanas, $\mathrm{y}$ en aquellos wild type con actividad elevada $(>55 \mathrm{nmol} / \mathrm{gHb} / \mathrm{h})$ debiera llegarse a dosis de 2,5-3 mg/kg/día $\mathrm{a}^{10,25,27}$.

Cuatro de 184 sujetos wild type (2,2\%) tuvieron actividad bajo $25 \mathrm{nmol} / \mathrm{gHb} / \mathrm{h}$ y uno de 16 heterocigotos $(6,25 \%)$ tuvo actividad normal. La existencia de individuos wild type pero con actividad enzimática bajo lo normal se puede explicar por varias razones: a) existencia de otras mutaciones para el gen TPMT diferentes a las analizadas, ya que las variantes estudiadas dan cuenta sólo de $95 \%$ de las enzimas con actividad deficiente ${ }^{28}$; b) otras variantes genéticas, como repeticiones en tándem de número variable (VNTR) en la región del promotor del gen de la TPMT, que se relacionan con baja actividad enzimática ${ }^{29,30}$ y c) variaciones funcionales o genéticas en otras enzimas implicadas en la metabolización de estos fármacos, distinta de la TPMT ${ }^{31-33}$. La existencia de un individuo heterocigoto TPMT ${ }^{\star} 3 \mathrm{~A}$ con actividad normal podría explicarse porque los puntos de corte de la actividad enzimática en nuestra población son ligeramente más bajos que en otras poblaciones ( 25 en vez de 28$)^{22,23}$.

La genotipificación y/o fenotipificación de TPMT es necesaria desde el punto de vista de efectividad y seguridad en el uso de estos fármacos, en especial para aquellos pacientes que inician el tratamiento con tiopurínicos. En estudios internacionales la determinación de la TPMT es costo efectiva en evitar efectos adversos graves como la mielotoxicidad $^{31,33}$. Aunque no existe consenso sobre cuál de los dos métodos implementar, probablemente son complementarios y es necesario un estudio de costo efectividad local para recomendar cuál es más conveniente, ya que ambos poseen ventajas y desventajas ${ }^{10,31,33}$. Además, la experiencia y nivel de implementación de cada centro en una de las técnicas (PCR-RFLP o HPLC) puede ayudar en la decisión.

La discordancia entre genotipo y fenotipo, asociado al hecho que la determinación de la TPMT no es tan certera en predecir una mielosupresión tardía y que este no es el único efecto adverso, hacen necesario mantener las precauciones y vigilancias periódicas universales mediante controles clínicos, de hemograma y función hepática.
En resumen, los individuos chilenos hispanos presentan un genotipo y fenotipo similar a la de otras poblaciones caucásicas, lo que apoya el uso racional de las tiopurinas de acuerdo a las recomendaciones internacionales manteniendo la vigilancia y precauciones conocidas. La implementación del estudio genotípico y/o fenotípico de la TPMT es factible y puede proporcionar una información útil al médico en la indicación de los tiopurínicos.

\section{Referencias}

1. Dubinsky MC. Azathioprine, 6-Mercaptopurine in Inflammatory Bowel Disease: Pharmacology, Efficacy, and Safety. Clin Gastroenterol Hepatol 2004; 2: 731-43.

2. Mc leod HL, Siva C. The thiopurine S-methiltransferase gene locus-implication for clinical pharmacogenomics. Pharmacogenomics 2002; 3 (1): 89-98.

3. Ohno K, Masunaga Y, Ogawa R, Hashiguchi M, Ogata H. A systematic review of the clinical effectiveness of azathioprine in patients with ulcerative colitis. Yakugaku zasshi Journal of the Pharmaceutical Society of Japan 2004; 124 (8): 555-60.

4. Pearson DC, May GR, Fick G, Sutherland LR. Azathioprine for maintaining remission of Crohn's disease. Cochrane Database Syst Rev 2000; (2): CD000067. Disponible en : www.thecochranelibrary.com

5. Chang JC, Cohen RD. Medical management of severe ulcerative colitis. Gastroenterol Clin N Am 2004; 33: 235-50.

6. Czaja AJ. Diagnosis and Treatment of Autoimmune Hepatitis. Hepatology 2002; 36 (2): 479-97.

7. Gaffney K, Scott DG. Azathioprine and Cyclophosphamide in the treatment of Rheumatoid Arthritis. Br J Rheumatol 1998; 37: 824-36.

8. Ioannou Y, Isenberg DA. Current concepts for the management of systemic lupus erythematosus in adults: a therapeutic challenge. Postgrad Med J 2002; 78: 599-606.

9. Tassaneeyakul W, Srimarthpirom S, Reungjui S, Chansung K, Romphruk A, Tassaneeyakul W. Azathioprineinduced fatal myelosuppression in a renal-transplant recipient who carried heterozygous $\mathrm{TPMT}^{\star} 1{ }^{\star} 3 \mathrm{C}$. Transplantation 2003; 76 (1): 265-6.

10. Ho GT, Lees C, Satsangi J. Pharmacogenetics and Inflammatory Bowel disease, progress and prospects. Inflamm Bowel disease 2004; 10: 148-58.

11. Remy C. S-Methylation with S-Adenoylmethionine Transmethylase and Catabolism in Mammalian tissues. J Biol Chem 1963; 238 (3): 1078-84. 
12. Weinshilboum RM, Sladeck SL. Mercaptopurine pharmacogenetics: monogenic inheritance of erythrocytes thiopurine methyltransferase activity. Am J Hum Genet 1980; 32: 651-62.

13. Salavaggione OE, Wang L, Wiepert M, Yee Vc, Weinshilboum RM. Thiopurine S-methyltransferase pharmacogenetics: variant allele functional and comparative genomics. Pharmacogenet Genomics 2005; 15: 801-15.

14. Gearry RB, Barclay ML. Azathioprine and 6-mercaptopurine pharmacogenetics and metabolite monitoring in inflammatory bowel disease. J Gastroenterol Hepatol 2005; 20: 1149-57.

15. Yates CR, Krynetski EY, Loennechen T, Fessing MY, Tai $\mathrm{HL}$, Pui $\mathrm{CH}$, et al. Molecular diagnosis of thiopurine-smethyltransferase deficiency: genetic basis for azathioprine and mercaptopurine intolerance. Ann Intern Med 1997; 126: 608-14.

16. Weinshilboum R. Inheritance and drug response. N Engl J Med 2003; 348: 529-37.

17. Laróvere LE, Dodelson de Kremer R, Lambooy LHJ, De Abreu RA. Genetic polymorphism of thiopurine Smethyltransferase in Argentina. Ann Clin Biochem 2003; 40: 388-93.

18. Isaza C, Henao J, López AM, Cacabelos R. Allelic variants of the thiopurine methyltransferase (TPMT) gene in the Colombian population. Methods Find Exp Clin Pharmacol 2003; 25 (6): 423-9.

19. Matson GA, Sutton HE. Distribution of hereditary blood groups among Indians in South America. IV. In Chile. Am J Phys Anthropol 1970; 27: 157-94.

20. Valenzuela CY, Acuña MP, Harb Z. [Sociogenetic gradient in the Chilean Population]. Rev Med Chile 1987; 155: 295-9.

21. Bernstein F. Die geographische Verteilung der Blutgruppen und ihre anthropologische Bedeutung. In: Instituto Poligrafico dello Stato, Eds, Comitato Italiano per lo Studio dei Problemi Della Populazione. Rome, Italy. 1931, p: 227.

22. Ford LT, Berg JD. Determination of thiopurine S-methyltransferase activity in erythrocytes using 6-thioguanine as substrate and a non-extraction liquid chromatographic technique. J Chromatogr B 2003; 798: 111-5.

23. Ford LT, Cooper SC, Lewis MJV, Berg JD. Reference intervals for thiopurine S-methyltransferase activity in red blood cells using 6-thioguanine as substrate and rapid non-extraction liquid chromatography. Ann Clin Biochem 2004; 41: 303-8.

24. Corominas H, Domenech M, Del Río E, Gich I, Domingo P, Baiget. Frequency of thiopurine S-methyltransferase alleles in different ethnic groups living in Spain. Med Clin 2006; 126 (11): 410-2.

25. Carter MJ, Lobo AJ, Travis S. Guidelines for the management of inflammatory bowel disease in adults. Gut 2004; 53 (Suppl V): v1-v16.

26. Egan L, Derijks LJ, Hommes DW. Pharmacogenomics in inflammatory bowel disease. Clin Gastro \& Hepatol 2006; 4: 21-8.

27. Meggitt SJ, Gray JC, Reynolds NJ. Azathioprine dosed by thiopurine methyltransferase activity for moderateto-severe atopic eczema: a double-blind, randomised controlled trial. Lancet 2006; 367 (9513): 839-46.

28. Schaeffeler E, Stanulla M, Greil J, Schrappe M, Eichelbaum M, Zanger UM, et al. A novel TPMT missense mutation associated with TPMT deficiency in a 5-yearold boy with ALL. Leukemia 2003; 17:1422-4.

29. Spire-Vayron DLM, Debuysere H, Fazio F, Sergent E, Bernard C, Sabbaqh N, et al. Characterization of a variable number tandem repeat region in the thiopurine S-methyltransferase gene promoter. Pharmacogenetics 1999; 9: 189-98.

30. Yan L, Zhang S, Eiff B, Szumlanski CL, Powers M, O’Brien JF, et al. Thiopurine methyltransferase polymorphic tandem repeat: genotype-phenotype correlation analysis. Clin Pharmacol Ther 2000; 68: 210-9.

31. Winter J, Walker A, Shapiro D, Gaffney D, Spooner RJ, Mills PR. Cost-effectiveness of thiopurine methyltransferase genotype screening in patients about to commence azathioprine therapy for treatment of inflammatory bowel disease. Aliment Pharmacol Ther 2004; 20: 593-9.

32. Baker DE. Pharmacogenomics of Azathioprine and 6-Mercaptopurine in Gastroenterologic Therapy. Rev Gastroenterol Disord 2003; 3 (3): 150-7.

33. Heckmann JM, Lambson EM, Little F, Owen EP. Thiopurine methyltransferase (TPMT) heterozygosity and enzyme activity as predictive tests for the development of azathioprine-related adverse events. J Neurol Sci 2005; 231: 71-80. 